\title{
A FIXED POINT THEOREM FOR SIMPLICIAL COMPLEXES WHOSE FUNDAMENTAL GROUPS ARE FREE PRODUCTS
}

\author{
FRANK RAYMOND
}

\begin{abstract}
A fixed point theorem for actions of toral and finite $p$-groups is obtained for finite simplicial complexes satisfying certain conditions on their fundamental groups and their universal covering spaces. Examples are constructed relating this result to other well-known results.
\end{abstract}

1. Introduction. Fixed point theorems have long been regarded as a very important part of the theory of group actions. Most fixed point theorems for general spaces derive from the Smith theory which is homological in nature. In this note we shall show, for finite simplicial complexes, how a fundamental group which is a nontrivial free product forces the existence of fixed points for toral and $p$-group actions.

The role of the fundamental group in the study of group actions on manifolds and nice topological spaces has become increasingly important. Of course, it is crucial for aspherical manifolds because their homotopy type is determined solely by the fundamental group. For example, if $M$ is a closed aspherical manifold and $G$ is a compact Lie group of homeomorphisms, then:

1. $G$ is a $k$-torus where $k \leqslant r=$ rank of center $\pi_{1}(M)$, if $G$ is connected [C-R-2].

2. In particular, if $r=0, G$ is finite [C-R-2].

3. If $r=0$, the torsion of the outer automorphism group of $\pi_{1}(M)$ is trivial, then $G$ itself must be trivial [C-R-1].

These results have been generalized and extended in many directions by various authors: R. Schultz, S. Schwartzman, M. C. and H. T. Ku, Asadi-Burghelea, W. Browder and W. C. Hsiang, R. Schoen and S. T. Yau, and K. B. Lee to name just several.

The fundamental groups of closed aspherical manifolds are indecomposable while those of connected sums are free products. Connected sums usually destroy asphericity and the fundamental group no longer dictates the topology of the sum. However, connected sums and free products still play a significant role, for example, in showing that in each arbitrary bordism class one may find closed manifolds without circle actions [C-R-3, 3.7], in E. Bloomberg's construction of closed nonaspherical 4-manifolds which admit no finite group actions [B], and in R. Schultz' study of hypertoral actions [S].

Received by the editors November 8, 1982.

1980 Mathematics Subject Classification. Primary 57S10, 57S17, 54H25; Secondary 20E06.

Key words and phrases. Transformation groups, covering spaces, ends, Freudenthal compactification, clc, cohomology groups, simplicial complex, fundamental group, free products, fixed points.

'Supported in part by the National Science Foundation. 
The theorems that we shall demonstrate here combine free products with certain homological assumptions to yield the existence of fixed points.

Let $X$ be homeomorphic to a connected finite simplicial complex, and let $Y^{\wedge}$ denote the 1-point compactification of the universal covering $Y$ of $X$. Assume the following:

(i) $\pi_{1}(X)=A * B$, where $A$ and $B$ are not trivial and both are not isomorphic to $\mathbf{Z} / 2 \mathbf{Z}$.

(ii) $H^{p}\left(Y^{\wedge} ; \mathbf{Q}\right)$ is finite dimensional, for all $p \geqslant 2 . H(-, \mathbf{Q})$ denotes the Čech cohomology functor with coefficients in the rational numbers $\mathbf{Q}$.

THEOREM 1. If $\phi: T^{s} \times X \rightarrow X$ is an action of the s-torus on $X$, then the set of fixed points, $F=X^{T^{s}}$, is not empty.

The proof has 2 main parts. The first step is to lift the action of $T^{s}$ to $Y$ and extend this lifted action to the Freudenthal end point compactification $Y^{*}$ of $Y$. By construction we know quite a bit about the fixed point set in $Y^{*}$, and we may combine this information, hypotheses (i) and (ii), and standard cohomological results to conclude that the original action has a nonempty fixed point set. Details appear in the next section where we also weaken the hypotheses (i) to the condition that the number of ends of $\pi_{1}(X, x)$ is infinite.

The extendability of group actions to Freudenthal compactifications was proved by the author some time ago, and a form of this result appears in [B, 2.3.1]. This extension result will be used more than once in the paper, so we shall give a precise statement below; the argument in [B] applies to the version stated below. The reader may consult [R-1] for the formulation of the Freudenthal compactification that we use here.

Lemma. Let $Y$ be a connected, locally connected, locally compact Hausdorff space and $Y^{*}$ denote its Freudenthal (end point) compactification and $i: Y \hookrightarrow Y^{*}$ the natural inclusion. Let $\phi: G \times Y \rightarrow Y$ be the action of a topological group on $Y$. Then there exists a unique extension $\phi^{*}: G \times Y^{*} \rightarrow Y^{*}$ to an action on $Y^{*}$ so that

$$
\begin{array}{ccc}
G \times Y & \stackrel{\phi}{\rightarrow} & Y \\
1 \downarrow \times i & & \downarrow i \\
G \times Y^{*} & \stackrel{\phi^{*}}{\rightarrow} & Y^{*}
\end{array}
$$

commutes.

In $\$ 3$ we shall prove our analogue of Theorem 1 for $p$-groups instead of toral groups.

Finally, in the last section we shall describe some examples which elucidate how (i) and (ii) are used in the proofs of Theorems 1 and 2. These examples then lead to a discussion about topological properties inherited by fixed point sets from the ambient space.

ACKNOWLEDGEMENTS. The referee suggested a complete reorganization of the presentation in the original manuscript. I have incorporated the suggestions in this 
revision and I gratefully acknowledge and appreciate these contributions to the present exposition.

I would also like to thank Reinhard Schultz for his interest and comments on the material in this note.

2. Proof of Theorem 1. We shall use a more general hypothesis than (i), namely,

(i) ${ }^{\prime} \pi_{1}(X)$ has an infinite number of ends.

(A well-known theorem of $\mathrm{H}$. Hopf states that a finitely presented group has $0,1,2$ or an infinite number of ends, see, for example [E]. Finite groups have 0 ends, finite extensions of $\mathbf{Z}$ have 2 ends, and nontrivial free products (where not both factors are $\mathbf{Z} / 2 \mathbf{Z}$ ) have an infinite number of ends. J. Stallings has determined when a finitely presented group $G$ has exactly an infinite number of ends ( $G=A_{C}^{*} B$ or $A_{C}^{*}$ where the amalgamating subgroup is finite and $G$ is not a finite extension of $\mathbf{Z}$ [St]). All other groups have one end.)

The first step in our argument is to lift the action $\phi: T^{s} \times X \rightarrow X$ to an action $\tilde{\Phi}$ : $T^{s} \times Y \rightarrow Y$ which commutes with the group of covering transformations.

Let us recall from [C-R-2, 4.3] that to lift the action of a path connected topological group $G$ on an arbitrary path connected space $X$ to its universal covering space it is necessary and sufficient that the image of the evaluation homomorphism $\mathrm{ev}_{*}^{x}: \pi_{1}(G, e) \rightarrow \pi_{1}(X, x)$ be trivial. Here $\operatorname{ev}^{x}(g)=g(x)$. We also note that image $\operatorname{ev}_{*}^{x}\left(\pi_{1}(G, e)\right)$ is always a central subgroup of $\pi_{1}(X, x)$, "independent" of the choice of base point $x$.

For the situation at hand we claim that $\mathrm{ev}_{*}^{x}: \pi_{1}\left(T^{s}, 1\right) \rightarrow \pi_{1}(X, x)$ has image a finite central subgroup of $\pi_{1}(X, x)$. This is a consequence of:

A finitely presented group with an infinite number of ends has no central elements of infinite order.

For, if the center of $\pi_{1}$ contains an element $g$ of infinite order, then the infinite cyclic subgroup generated by $(g)$ is normal in $\pi$. If the quotient is finite then $\pi$ has 2 ends. If the quotient is infinite then $\pi$ has one end (see [L-R, Lemma 4 or Cohen, Proposition 2.5]). Therefore, each element of the center has finite order.

Now let ' $T$ s be a finite covering group of $T^{s}$ so that

$$
\pi_{1}\left(T^{s}, 1\right) \stackrel{\rho^{*}}{\rightarrow} \pi_{1}\left(T^{s}, 1\right) \stackrel{\mathrm{ev}_{*}^{x}}{\rightarrow} \pi_{1}(X, x)
$$

has trivial image, where $\rho:{ }^{\prime} T^{s} \rightarrow T^{s}$ is the covering projection. Then ' $T$ s acts on $X$ via $\rho$ and $X^{T^{s}} \neq \varnothing$ if and only if $X^{\prime T^{s}} \neq \varnothing$. Therefore, we may as well assume image $\left(\mathrm{ev}_{*}^{x}\left(\pi_{1}\left(T^{s}, 1\right)\right)\right)$ is trivial.

Lift the action $\phi: T^{s} \times X \rightarrow X$ to an action $\tilde{\phi}: T^{s} \times Y \rightarrow Y$ on the universal covering $Y$ of $X$. It commutes with the covering transformations and $T_{y}^{s} \subset T_{z}^{s}$, for each $y \in Y$ that lies over a $z \in X$. Thus, if $T_{y}^{s}=T^{s}$, then $z$ must be a fixed point of $X$. Therefore, we may assume $T_{v}^{s} \neq T^{s}$, for all $y \in Y$ or else there is nothing to prove.

We extend the action of $T^{s}$ on $Y$ to $Y^{*}$, the Freudenthal compactification of $Y$ by the Lemma in $\S 1$. Now $B=Y^{*}-Y$, which is a Cantor set since $\pi_{1}(X)$ has an infinite number of ends, is $T^{s}$-invariant. Hence, $B=\left(Y^{*}\right)^{T^{s}}$. By (ii), we know that 
$H^{p}\left(Y^{*} ; \mathbf{Q}\right)$ is finite dimensional for all $p \geqslant 2$. By [R-1, 1.13], we may easily deduce that $H^{1}\left(Y^{*} ; \mathbf{Q}\right)=0$. Thus, $H^{*}\left(Y^{*} ; \mathbf{Q}\right)$ is finite dimensional. Therefore, $H^{*}\left(Y^{*} T^{s} ; \mathbf{Q}\right)$ must be finite dimensional by [F or $\mathbf{C}]$. This yields a contradiction.

Alternatively, we may use the fact that the finite dimensionality of $H^{*}\left(Y^{*} ; \mathbf{Q}\right)$ and the clc $_{\mathbf{Q}}$ property of $Y$ implies that $Y^{*}$ is $\operatorname{clc}_{\mathbf{Q}}$ by $[\mathbf{R}-\mathbf{1}, 4.4]$. Hence, $Y^{*} S^{\prime}=B$ would have to be $\operatorname{clc}_{\mathbf{Q}}$ by $[\mathbf{F}]$. Since $B$ is not even locally connected we have an alternative contradiction.

3. The theorem for $p$-groups. We shall let $p$ be a prime $\geqslant 2$ and shall assume exactly the same hypothesis as in 1.1 except we replace (ii) by

(ii- $p$ ) $H^{j}\left(Y^{\wedge} ; \mathbf{Z} / p \mathbf{Z}\right)$ is finite dimensional, for all $j \geqslant 2$.

The action induced on the fundamental group by a toral action is always trivial. We then should also expect to assume its analogue if we wish to obtain a result similar to 1.1 for $p$-groups.

THEOREM 2. Let $\phi: G \times X \rightarrow X$ be an action of a finite p-group on $X$ which satisfies (i) and (ii- $p$ ). If the homomorphism $\Psi: G \rightarrow \operatorname{Out}(A * B)$ induced by the action on $X$ is trivial, then the set of fixed points, $F=X^{G}$, is not empty.

Proof. Recall that $\psi$ is simply the composite $G \stackrel{\text { inc. }}{\rightarrow}$ Homeomorphisms of $X \stackrel{\eta}{\rightarrow}$ Out $\pi_{1}(X, x)$. Each homeomorphism induces an automorphism of $\pi_{1}(X, x)$ which is uniquely determined up to an inner automorphism since we have no control of base points. This assignment $\eta$ is a homomorphism. The group $\operatorname{Out}(A * B)$ is the automorphism group of $(A * B)$ modulo the group of inner automorphisms.

If we now take the group of liftings of the $G$ action to $Y$, the universal covering of $X$, we get a group extension

$$
1 \rightarrow \pi_{1}(X, x) \rightarrow E \rightarrow G \rightarrow 1 .
$$

Since $\psi$ is trivial and $\pi_{1}(X, x)$ is centerless, $E$ is $\pi_{1}(X, x) \times G$. Thus the action of $G$ lifts to a $G$ action on $Y$ that commutes with the group of covering transformations. A specific description of this lifting can be found in [C-R-1, 1.2]. The ends of $\pi_{1}(X, x)$, and hence of $Y$, can be described by sequences of elements from $\pi_{1}(X, x)$. These sequences are acted upon by the elements of $G$. Each $g$ represents conjugation by some element, say $\alpha_{g} \in \pi_{1}(X, x)$. This lift multiplies a sequence by $\alpha_{g}$. But the end is only determined by the "last" term of the sequence and so the end must be left fixed by the action of $g \in G$. Now the method employed in the proof of Theorem 1 applies.

4. Some examples. It is well known $[\mathrm{C}]$ that the Euler characteristic $\chi(X)=$ $\chi\left(X^{T^{s}}\right)$, and consequently, $\chi(X) \neq 0$ implies $X^{T^{s}} \neq \varnothing$. Therefore, Theorems 1 and 2 are new only when $\chi(X)=0$ (or $\chi(X) \equiv 0(p)$ ). Also, as one would expect, the theorems may fail without assumption (ii).

The first class of examples in 4.1 illustrate the theorems for 3-dimensional manifolds. In these examples one can actually "see" the lifted actions and completely understand the extensions to the Freudenthal compactifications. 
In 4.2 below we shall modify the examples above to construct 6 -dimensional simplicial complexes $S$ with the following properties:

(i) $S$ has a circle action with no fixed points.

(ii) This action lifts and extends to the Freudenthal compactification $\tilde{S}^{*}$.

(iii) $H^{4}\left(\tilde{S}^{\wedge} ; \mathbf{Q}\right)$ is infinite dimensional.

(iv) $\tilde{S}^{*}$ is locally connected, but the circle action's fixed point set $B=\tilde{S}^{* S^{1}}$ is not ( $B$ is also the set of ends of $\tilde{S}$ ).

The proof of the fixed point Theorems 1 and 2 depend crucially upon the facts that finite dimensionality of the cohomology groups or the cohomological local connectivity $\left(\mathrm{Clc}_{\mathbf{Q}}\right)$ properties are inherited by the set of fixed points from the ambient space. On the basis of these cohomological results one might expect to be able to weaken the finite dimensionality or clc assumptions and still obtain weakened inheritance on the set of fixed points. For example, perhaps local connectedness is inherited by the fixed point set of a "reasonably nice" torus action. However, the examples below show that such conjectures are false. Another construction making this point, which is due to Morton Brown, is given in 4.5.

4.1. Let $L$ be any 3-dimensional lens space so that $\left|\pi_{1}(L)\right|>2$. The circle group acts effectively on $L$ with a circle of fixed points, one exceptional orbit, and all other orbits are principal. (Take a rotation about the core of one solid torus and sew it equivariantly to a Seifert fibered torus. With the correct choice of Seifert fibered torus one can make any lens space.) Choose $x \in L^{S^{\prime}}$ and take an $S^{1}$-invariant 3-cell neighborhood. Now with 2 copies of $L$ (or even a copy of $L$ and of $L^{\prime}$ ) form the equivariant connected sum, $R=L \# L$ using the invariant 3-cells to form the connected sum. We obtain a new oriented 3 manifold with an $S^{1}$-action having a circle of fixed points and two exceptional orbits. $\pi_{1}(R)=\pi_{1}(L) * \pi_{1}(L)$.

Since we have fixed points the action may be lifted to the universal covering $\tilde{R}$ which is diffeomorphic to $\left\{\mathbf{R}^{3}-\right.$ (a totally disconnected set-in fact a Cantor setremoved from the " $z$-axis") $\}$. The lifted action is just rotation about the $z$-axis $[\mathbf{R}-2$, Proposition 4]. The Freudenthal end point compactification $\tilde{R}^{*}$ of $\tilde{R}$ just inserts the missing points along the $z$-axis (and $\infty$ ) to yield the 3-sphere, $S^{3}$. Observe that the $S^{1}$-action naturally extends to $S^{3} \cdot H^{p}\left(S^{3} ; \mathbf{Q}\right) \cong H_{c}^{p}(\tilde{R} ; \mathbf{Q})=H^{p}\left(\tilde{R}^{\wedge} ; \mathbf{Q}\right) \cong$ $H^{p}\left(\tilde{R}^{*} ; \mathbf{Q}\right)$, for $p>1$, which certainly is finite dimensional. The fixed point set of the $S^{1}$-action on $\tilde{R}^{*}=S^{3}$ is a circle and its cohomology is finite dimensional. Moreover, $\tilde{R}^{*}=S^{3}$ is $\operatorname{clc}_{\mathbf{Q}}$ and this property is inherited by the fixed point set. (Note that the group $H^{1}\left(\tilde{R}^{\wedge} ; \mathbf{Q}\right)$ is infinite dimensional.)

4.2. In contrast to 4.1 we shall construct a 6-dimensional simplicial complex $S$ so that $\pi_{1}(S)=\pi_{1}(R)$ with an $S^{1}$-action without fixed points. Take the same $R$ as above and form $R \times S^{3}=(L \# L) \times S^{3} \equiv S$. For our $S^{1}$-action we take the diagonal action which is trivial on the first $(L \# L)$ factor and the standard free Hopf-action on the $S^{3}$-factor.

Since $\pi_{1}(S)=\pi_{1}\left((L \# L) \times S^{3}\right)=\pi_{1}(L) * \pi_{1}(L)$ has trivial center, this free $S^{1}$ action lifts to the universal covering $\tilde{S}=\tilde{R} \times S^{3}$ and commutes with the covering transformations. Extend the action of $S^{1}$ to $\tilde{S}^{*}$, the Freudenthal compactification of $\tilde{S}$. Now $\tilde{S}^{S^{1}}$ is empty for otherwise $S^{1}$ would have fixed points on $S$. But, $\tilde{S}^{*}-\tilde{S}$ 
must be fixed. Since $\pi_{1}(S)=\pi_{1}(L) * \pi_{1}(L), B$ is a Cantor set-in fact, the "same" Cantor set appearing in 4.1. $H^{*}(B ; \mathbf{Q})$ is infinite dimensional. Moreover $B$ is not locally connected even though $\tilde{S}^{*}$ is locally connected. We observe that $H^{4}\left(\tilde{S}^{\wedge} ; \mathbf{Q}\right)$ is not finite dimensional.

Actually, with a little more effort 3-dimensional examples utilizing just 2 copies of $L$ in 4.1 can also be used to construct examples similar to that just described.

4.3. 4.2 is just a special case of the following general construction. Replace $S^{3}$ by any finite connected complex $W$ admitting an action of $S^{1}$ without fixed points and with $\pi_{1}(W)$ finite. Replace $L \# L$ by any finite connected complex $R$ whose fundamental group has more than 2 ends. $S^{1}$ acts on the product $R \times W=S$ by just acting on the second factor and so has no fixed points. After possibly passing to a finite cover of $S^{1}$, the action is lifted to the universal covering $\tilde{S}$ and extended to the Freudenthal compactification, $\tilde{S}^{*}$. Again $B$ is the fixed point set and $H^{k+1}\left(\tilde{S}^{\wedge} ; \mathbf{Q}\right)$ cannot be finite dimensional because $H_{c}^{\mathrm{l}}(\tilde{R} ; \mathbf{Q})$ is not finite dimensional, and $H^{k}(W ; \mathbf{Q})$ is not trivial for some $k>0$.

4.4. We obtain the same constructions and the analogous conclusions of $4.1,4.2$ and 4.3 by choosing a cyclic group of prime order $p$ inside the circle group. (Take $p$ so that it does not divide the orders of the exceptional orbits in 4.1 and those which might appear in 4.3.)

4.5. Morton Brown suggested another construction for the violation of local connectedness.

Take any compactum $A$ in $I^{n}$, the standard $n$-cell. In $\mathbf{R}^{n+1}$, form $X^{+}=\{(x, d) \mid x$ $\in I^{n} \subset \mathbf{R}^{n} \times 0$, and $\left.\rho(x, A)=d\right\}$ where $\rho$ is the Euclidean distance in $\mathbf{R}^{n} \subset \mathbf{R}^{n} \times \mathbf{R}^{1}$. Put $X^{-}=\left\{(x,-d) \mid(x, d) \in X^{+}\right\}$. On $X=X^{+} \cup X^{-}$, there is an obvious involution $(x, d) \leftrightarrow(x,-d)$ and $A=X^{\mathbf{Z} / 2 \mathbf{Z}}$ is the set of fixed points. $X^{+}$and $X^{-}$are both homeomorphic to $I^{n}$. It is easy to see that $X$ is always locally connected even though $A$ may not be.

Now $X$ behaves somewhat like the suspension of $A$. For example, $H^{p}(A) \approx$ $H^{p+1}(X), p \geqslant 0$ (using augmented cohomology in dimension 0 and the MayerVietoris sequence). Similarly, using the Mayer-Vietoris sequence we see that if $A$ is cohomologically locally connected at $a \in A$, in dimensions $-1,0,1,2, \ldots, k$, then $X$ is cohomologically locally connected at $a \in X$ in dimensions $-1,0, \ldots, k+1$. If $A$ is not $(k+1)$-cohomologically locally connected at $a \in A$, then neither is $X(k+2)$ cohomologically locally connected at $a \in X$.

Brown's construction can easily be modified by using an extra dimension to yield violations of inheritance of local connectedness for actions of cyclic $p$-groups or circle groups.

\section{REFERENCES}

[B] Edward M. Bloomberg, Manifolds with no periodic homeomorphisms, Trans. Amer. Math. Soc. 202 (1975), 67-78.

[Cohen] Daniel E. Cohen, Groups of cohomological dimension one, Lecture Notes in Math., vol. 245, Springer-Verlag, Berlin and New York, 1972.

[C] P. E. Conner, On the action of the circle group, Michigan Math. J. 4 (1957), 241-248. 
[C-R-1] P. E. Conner and Frank Raymond, Manifolds with few periodic homeomorphisms, Proc. Second Conf. Compact Transformation Groups, Lecture Notes in Math., vol. 299, Springer-Verlag, Berlin and New York, 1972, pp. 1-75.

[C-R-2] __ Actions of compact Lie groups on aspherical manifolds, Topology of Manifolds (Cantrell and Edwards, eds.), Markham, Chicago, Ill., 1970, pp. 227-264.

[C-R-3] _ Deforming homotopy equivalences to homeomorphisms in aspherical manifolds, Bull. Amer. Math. Soc. 83 (1977), 36-85.

[E] D. B. A. Epstein, Ends, topology of 3-manifolds (M. K. Fort, Jr., ed.), Prentice-Hall, Englewood Cliffs, N. J., 1962, pp. 110-117.

[F] E. E. Floyd, Fixed point sets of compact abelian Lie groups, Ann. of Math. (2) 66 (1957), 30-35.

[L-R] Ronnie Lee and Frank Raymond, Manifolds covered by Euclidean space, Topology 14 (1975), 49-57.

[R-1] Frank Raymond, The end point compactification of manifolds, Pacific J. Math. 10 (1960), 947-963.

[R-2] Classification of the actions of the circle on 3-manifolds, Trans. Amer. Math. Soc. 131 (1968), 51-78.

[S] Reinhard Schultz, Group actions on hypertoral manifolds. II, J. Reine Angew Math. 325 (1981), 75-86.

[St] J. R. Stallings, On torsion-free groups with infinitely many ends, Ann. of Math. (2) 88 (1968), 312-334.

Department of Mathematics, University of Michigan, Ann Arbor, Michigan 48109 